\title{
La restauración ecológica en México: una disciplina emergente en un país deteriorado
}

\section{Ecological restoration in Mexico: An emerging discipline in a deteriorated country}

\author{
Karina Calva-Soto' y Numa P. Pavón ${ }^{*}$
}

' Universidad Autónoma del Estado de Hidalgo. Centro de Investigaciones Biológicas. Pachuca, Hidalgo, México.

\section{RESUMEN}

Los ecosistemas terrestres se encuentran deteriorados por las actividades humanas, por lo que es urgente realizar proyectos de restauración para recuperar la biodiversidad y los servicios ambientales. Sin embargo, la restauración ecológica es aún incipiente en México, como un tema multidisciplinario que implica la generación de teoría y práctica. En este estudio, se analizaron las publicaciones sobre restauración ecológica en México, para brindar una visión del estado actual del conocimiento en el tema e identificar sus limitantes, retos y avances. Usando bases de datos, se obtuvieron 20764 referencias, de las cuales se seleccionaron 402 trabajos. Todas las referencias seleccionadas fueron revisadas y clasificadas considerando diversos criterios. Entre estos, las preguntas de investigación en restauración ecológica: ¿por qué, qué, cómo, dónde y cuándo restaurar? Del total, 32\% correspondieron a estudios de restauración ecológica sensu stricto. En ocho estados no se encontraron referencias de estudios; Chiapas y Veracruz fueron los más atendidos. La mayoría de los trabajos se realizaron en bosques tropicales (43\%) y templados (13\%). Solo 10\% de los trabajos incluyeron todas las preguntas de investigación. En México la restauración ecológica es una disciplina emergente, la cual, a pesar de las complicaciones políticas, económicas y sociales del país, ha recibido cada vez mayor atención por diferentes grupos de trabajo. Aunque se obtuvieron pocos estudios de restauración, comparados con el total global, se observó un incremento en los últimos diez años. En esta revisión se destaca la necesidad de generar equipos multidisciplinarios para cubrir con los aspectos necesarios en la generación de proyectos de restauración ecológica, dentro de sistemas socioecológicos dinámicos.

PALABRAS CLAVE: bosques templados, buscadores de Internet, ecología aplicada, ecología de la restauración, revisión bibliográfica, sucesión ecológica.

\section{ABSTRACT}

Terrestrial ecosystems are perturbed by human activities, and many of them need to be restored so biodiversity and environmental services can recover. However, ecological restoration is incipient in Mexico as a multidisciplinary issue that still requires further advances in theory and practices. Publications on ecological restoration in Mexico were analyzed to provide an insight into the current state of knowledge on the subject and identify gaps, biases, and progress. We used databases on the web, from which 20764 references were obtained and 402 were selected, which met all of the requirements. All selected references were reviewed and classified considering various criteria. Among these, research questions in ecological restoration: why, what, how, where and when to restore. From the total, $32 \%$ were studies of ecological restoration sensu stricto. In eight states no reference studies were found. Chiapas and Veracruz were the most cited. Most work was conducted in tropical (43\%) and temperate forests $(13 \%)$. Only $10 \%$ of the references included all restoration research questions. In Mexico, ecological restoration is an emerging discipline, which despite the political, economic and social complications of the country, has received increased attention by different working groups, principally in the last 10 years. It should be noted that so far, studies continue to have a focus on restoration ecology, it being necessary to develop strategies to help increase ecological restoration projects aiming to restore degraded ecosystems.

KEYWORDS: temperate forests, internet search engines, applied ecology, restoration ecology, literature revision, ecological succession.

\section{INTRODUCCIÓN}

México es un país ambientalmente deteriorado, donde $90.7 \%$ de la superficie nacional (177 642000 ha) presenta algún nivel de degradación (Comisión Nacional Forestal [Conafor] y
Universidad Autónoma Chapingo [UACh], 2013). Los ecosistemas de México han sido impactados por actividades humanas desde épocas prehispánicas, las cuales todavía continúan. La tasa de deforestación de $-0.2 \%$ (1990-2016) genera 
pérdidas anuales cercanas a 354000 ha (Organización de las Naciones Unidas para la Alimentación y la Agricultura [FAO], 2010; 2015). A pesar de la los avances en legislación ambiental para la conservación y protección de ecosistemas, las perturbaciones generadas por actividades humanas continúan (Carabias, Arriaga y Cervantes-Gutiérrez, 2007), incluso al interior de las áreas naturales protegidas (Porter-Bolland et al., 2011; Ceccon, Barrera-Cataño, Aronson y Martínez-Garza, 2015). Por esto, no solo se deben redoblar los esfuerzos de conservación, sino, además, desarrollar proyectos de restauración ecológica, que permitan recuperar servicios ecosistémicos y mantener la biodiversidad (Society for Ecological Restoration [SER], 2004).

Considerando el carácter multidisciplinario de la restauración, es complicado conjuntar acciones académicas, sociales y económicas, por lo que sigue siendo más práctico reforestar. Sin embargo, los programas de reforestación por lo general utilizan pocas especies, incluso exóticas, que pueden modificar adversamente las condiciones ambientales (Carabias et al., 2007; González-Espinosa et al., 2007). Por ejemplo, la Conafor lleva a cabo la producción de 62 especies de plantas para reforestación, siendo 11 las más importantes, destacándose Pinus gregii, $P$. cembroides, $P$. pseudostrobus, $P$. engelmannii y $P$. montequmae, usadas tanto en zonas templadas como semiáridas (López, 2016).

La restauración ecológica es un tema emergente, con escasos trabajos desarrollados (Armesto et al., 2007; Sarukhán, 2007; Bonfil, Fernández y Fernández y González-Espinosa, 2015; Ceccon et al., 2015). A pesar de lo anterior, a nivel mundial se ha incrementado el interés en el tema. La creación en el 2004 de la SER, el incremento en el número de publicaciones y las revistas especializadas en el tema son prueba de ello. En particular, se destacan las revistas Restoration Ecology y Ecological Restoration; esta última dedicó en 2010 un número especial sobre trabajos de restauración ecológica en México. Previamente, el Boletín de la Sociedad Botánica de México, dedicó en 2007 el número 80 a publicar trabajos sobre restauración ecológica en el país. Además, en el 2014 se realizó el Primer Simposio Mexicano de Restauración Ecológica, donde se plantearon las necesidades de investigación para el desarrollo de la restauración ecológica (Ceccon et al., 2015). La mayoría de los trabajos presentados en este evento fueron realizados para un número reducido de tipos de vegetación, tales como las selvas tropicales estacionalmente secas.

Es importante destacar que diversos estudios de ecología, se han utilizado como fuente de información aplicable para la restauración ecológica, por ejemplo: banco, lluvia y dispersión de semillas, establecimiento de plantas, interacciones bióticas y sucesión ecológica, entre otros. Dado que estos estudios brindan las bases científicas y metodológicas aplicables, pueden ser catalogados dentro del campo de la ecología de la restauración (SER, 2004). Mientras que la restauración ecológica sensu stricto tiene como objetivo la práctica de la restauración de ecosistemas en proyectos específicos (Perring et al., 2015), con la meta de involucrar temas sociales, económicos, jurídicos y políticos desde la perspectiva transdisciplinaria (Carabias et al., 2007). Tomando en cuenta estas diferencias, el número de trabajos de restauración ecológica sensu stricto en México puede ser considerado como reducido.

\section{OBJETIVOS}

El objetivo principal de este trabajo fue analizar el desarrollo de las investigaciones sobre restauración ecológica en México, mediante una revisión bibliográfica exhaustiva (1999 a 2016) para brindar una visión del estado actual del conocimiento en el tema e identificar sus sesgos, limitantes y avances.

\section{MATERIALES Y MÉTODOS}

Se realizó una búsqueda exhaustiva de publicaciones sobre restauración ecológica de ecosistemas en México de 1999 a 2016. Para la revisión se incluyeron publicaciones (revistas, libros y capítulos de libro), reportes técnicos, memorias de congresos y memorias de un simposio. Se revisaron bases de datos y buscadores de información como ACS Publications, Alliance of Crop, Soil and Environmental Science Societies, Bione, Conabio, Dialnet, Ebsco, Emerald, Science Direct, Springer Link y Wiley. Se emplearon como palabras clave las siguientes: "ecological restoration + Mexico" y "restauración ecológica + México". Se descartaron referencias de trabajos realizados en Nuevo México, Golfo de México, ecosistemas acuáticos o que citaban trabajos de México, pero fueron realizados en otros países. En particular, se revisó el total de los contenidos de las revistas Restoration Ecology y Ecological Restoration. 
Se revisaron las memorias de los congresos de la Sociedad Científica Mexicana de Ecología (2006, 2008, 2011, 2013 y 2015) y el primer simposio de restauración ecológica en México (2014). En general las memorias se han catalogado como literatura gris, sin embargo, se consideró que aportaban información valiosa que frecuentemente se pierde al no publicarse. No se consideraron los resultados de estos trabajos, pero sí la información sobre el tipo de investigación, el sitio de estudio y el tipo de vegetación.

Posterior a la búsqueda de la literatura, el total de los trabajos fueron revisados y caracterizados. Los aspectos identificados de cada trabajo fueron: tipo de investigación, tipo de vegetación que se trabajó, año de publicación, estado de la república donde se realizó y objetivos, entre otros. La restauración ecológica, de acuerdo con sus objetivos, puede ser dividida en dos tipos de investigación: i) restauración ecológica sensu stricto (REs), que se refiere a la aplicación de diferentes aspectos y modelos teóricos en ecosistemas degradados y ii) ecología de la restauración (ER), que se enfoca en los estudios básicos que generan información científica, la cual podría ser utilizada para la restauración. En algunos casos un trabajo incluyo ambos tipos de investigación (A). Además, para cada referencia se identificaron los puntos relevantes del trabajo, las preguntas de investigación consideradas y sus respuestas. Esto último para aportar sugerencias en las áreas a fortalecer en futuros estudios sobre restauración ecológica. Para lo anterior, se aplicó la propuesta de Hobbs, Walker y Walker (2007), quienes consideraron que los trabajos de restauración deben contestar las siguientes preguntas.

¿Por qué restaurar? En este paso se decide si se requiere la restauración y por qué. Hace referencia a lo que se percibe antes de la restauración y lo que se espera observar a largo plazo.

- ¿Dónde restaurar? Se refiere al tipo de ecosistema o paisaje a restaurar, sus características, su etapa sucesional y el alcance espacial que se espera restaurar a largo plazo.

¿Qué restaurar? Toma en cuenta el objetivo o punto final que se espera obtener con la restauración; generalmente la información proviene de un "sistema de referencia", a partir de datos históricos, conocimiento de la estructura, riqueza de especies y aspectos del ambiente.

- ¿Cómo restaurar? Tiene que ver con la evaluación de los costos y beneficios, el control operacional, la evaluación técnica, las herramientas y los recursos necesarios para la restauración. También se consideró el uso de conocimientos tanto científicos como locales.

¿Cuándo restaurar? Considera el manejo físico durante la restauración, el acondicionamiento del sitio, el establecimiento de la vegetación, la revisión, el monitoreo y las intervenciones durante proyecto.

\section{Resultados}

Con la revisión de las bases de datos se obtuvieron 20764 referencias; sin embargo, muchas se encontraron repetidas. De todas estas, se seleccionaron 98, las cuales cumplieron con los criterios establecidos previamente. Además, se revisaron un total de 306 memorias de trabajos, presentados en congresos y simposios.

Fue notorio un incremento en el número de publicaciones en los últimos años. La mayoría corresponde a trabajos sobre ER, tanto en las publicaciones (68\%) (Fig. 1) como en los resúmenes de congresos (91\%). Los trabajos publicados, considerados propiamente como de RE, han sido escasos. Solo 6\% de las publicaciones incluyeron tanto objetivos de REs como de ER (Fig. 1) y 7\% en las memorias de congresos y simposios.

El número de revistas que publican estudios sobre restauración es amplio; para esta revisión se identificaron 26. Sin embargo, la mayoría de ellos se encontraron en dos: Restoration Ecology y Ecological Restoration (Fig. 2). Se obtuvieron 11 reportes técnicos y siete capítulos de libro.

En 19 estados del país se han realizado estudios de restauración (Fig. 3); sin embargo, se destacan Veracruz y Chiapas con 49\% de las publicaciones. Al incluir la información de los resúmenes de congresos, Morelos, Ciudad de México y Michoacán incrementaron notablemente su producción. Además, el número de estados se incrementó a 24, al incluir a Guanajuato, Nayarit, Querétaro, San Luis Potosí y Tabasco.

La mayoría de las publicaciones son de trabajos donde se estudió solo un tipo de vegetación; únicamente $6 \%$ estudiaron dos; 4\% estudiaron tres y $1 \%$, cuatro o más. Existió un sesgo hacia estudios en bosques tropicales $(37 \%$ en publicaciones y 45 $\%$ en memorias de congresos y simposios). Además, se destacaron los trabajos realizados en bosques templados $(16 \%$ en publicaciones y $12 \%$ en memorias de congresos y el simposio), 
Calva-Soto y Pavón. La restauración ecológica: una disciplina emergente en un país deteriorado

bosques mesófilos de montaña $(16 \%$ en publicaciones y $7 \%$ en memorias de congresos y el simposio) y vegetación árida y semiárida $(15 \%$ en publicaciones y $19 \%$ en memorias de congresos y el simposio). En general, los humedales se encontraron poco representados $(6 \%)$. Otro tipo de sitios donde se han realizado trabajos, pero que fueron poco representados, son suelos con cenizas volcánicas (3\%), cárcavas (1\%), dunas $(1 \%)$, islas $(1 \%)$ y cercas vivas $(1 \%)$. Las memorias de congresos y simposios agregan, además, pedregales $(3 \%)$, pastizales $(3 \%)$ y vegetación urbanizada $(0.8 \%)$ (Tabla 1$)$.

La mayoría de los estudios responden cuatro de las cinco preguntas que se propone deberían contestar las investigaciones sobre restauración (Fig. 4a). Se observó que hay un mayor número de publicaciones sobre REs que contestan cuatro preguntas, mientras que los de ecología de la restauración en su mayoría contestan tres o menos. De las cinco preguntas, la que más se tomó en cuenta en las investigaciones publicadas fue la relacionada con el desarrollo de técnicas, herramientas y conocimientos para la restauración (¿Cómo restaurar?) y la caracterización de las áreas de estudio (¿Dónde restaurar?) (Fig. 4b). Otras consideraciones importantes que gran parte de los estudios tomaron en cuenta fue el manejo físico durante la restauración y el monitoreo posterior (¿Cuándo restaurar?) (Fig. 4b). No fueron consideradas las memorias de congresos y simposios, debido a la escasa información que proporcionaban.

Derivado de la revisión se construyeron tres anexos. El primero es una tabla con información con las referencia completas de los trabajos publicados, información relevante, tipo de vegetación, sitio de estudio, tipo de estudio y las respuestas que se dan a las preguntas de investigación de acuerdo con Hobbs et al. (2007). En un segundo anexo se incluyen los títulos de los trabajos presentados en cinco de los Congresos Mexicanos de Ecología, que incluyen la información de los autores, institución, sitio de estudio, tipo de vegetación y tipo de estudio. Finalmente, en un tercero se incluye la información del anexo anterior, pero referente a los trabajos presentados en el primer Simposio Nacional de Restauración de Ecosistemas.

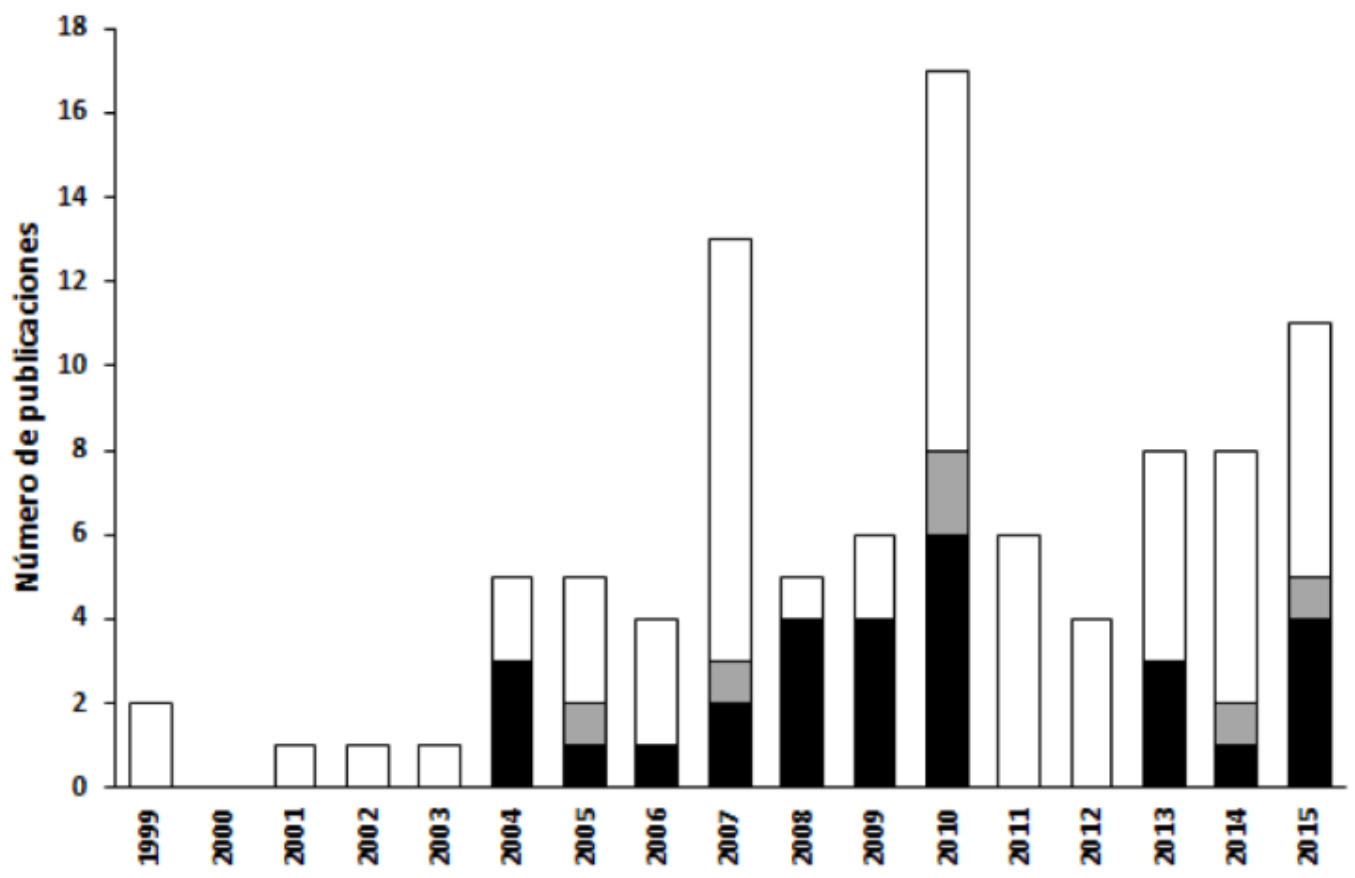

FIGURA 1. Número de trabajos publicados entre 1999 y 2015 sobre restauración ecológica en México.

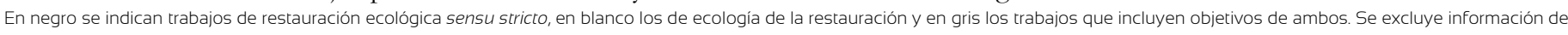
congresos y simposio. 


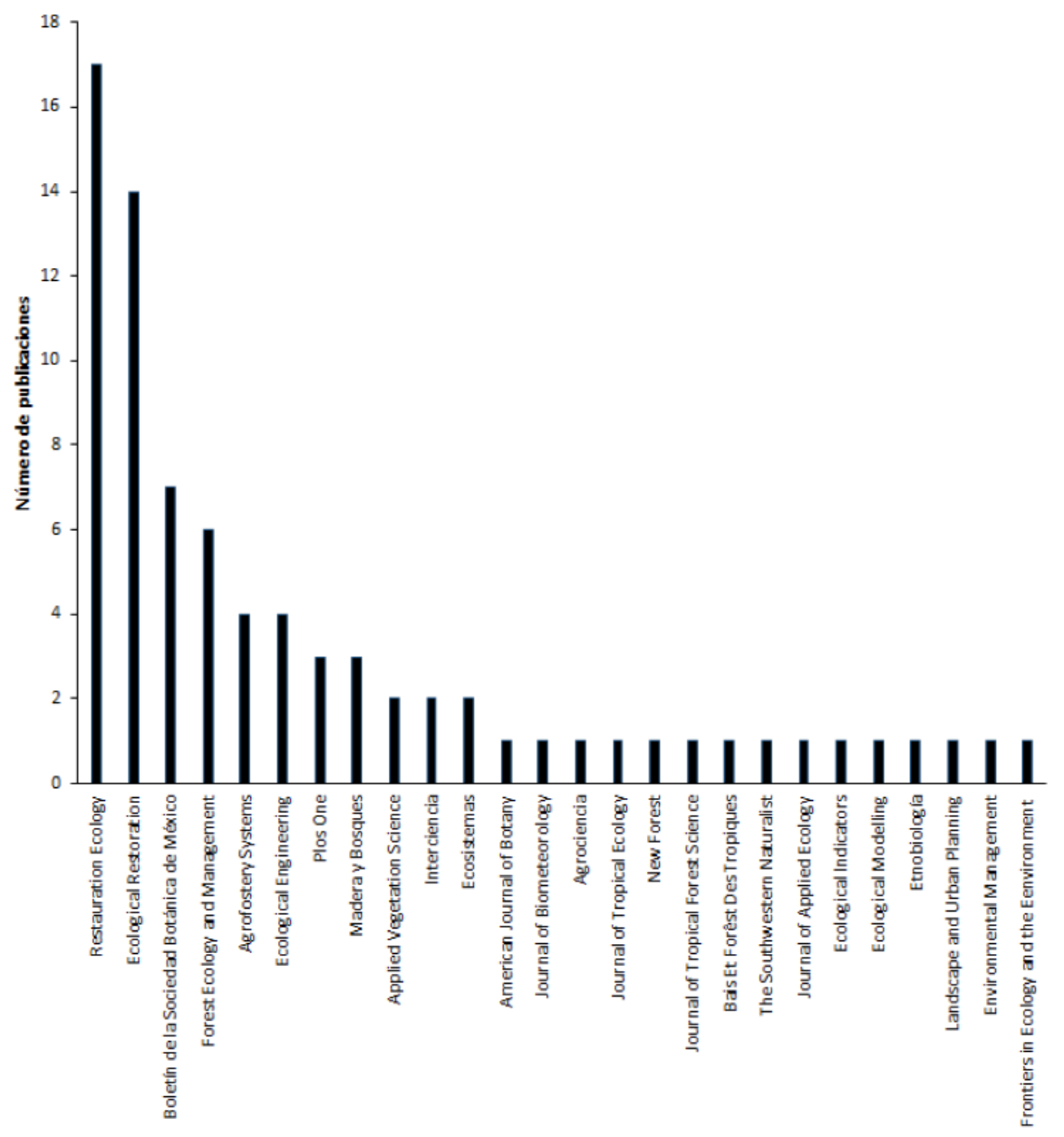

FIGURA 2. Número de trabajos publicados entre 1999 y 2016 en revistas indizadas internacionales.

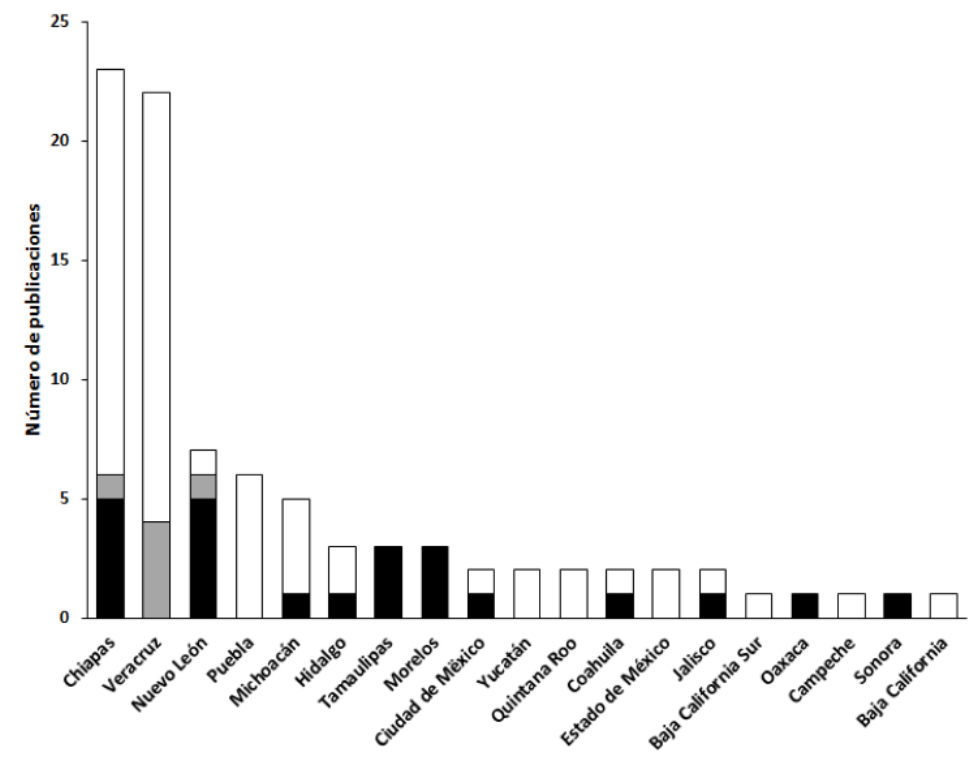

FIGURA 3. Número de trabajos publicados (1999-2016) en cada uno de los estados del país.

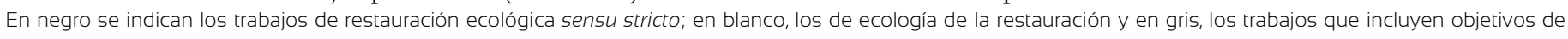
ambos. Se excluye información de congresos. 
Calva-Soto y Pavón. La restauración ecológica: una disciplina emergente en un país deteriorado

Tabla 1. Tipos de vegetación en donde se han realizado estudios de restauración ecológica en México y que hayan sido publicados (1999-2016).

\begin{tabular}{|c|c|c|}
\hline & Vegetación registrada en la literatura & $\begin{array}{l}\text { Número } \\
\text { publicaciones }\end{array}$ \\
\hline Bosques tropicales & $\begin{array}{l}\text { Dryland forests, Evergreen tropical rainforest, Lowland tropical high } \\
\text { evergreen rain forest, Lowland tropical rain forest, Seasonal tropical } \\
\text { forest, Selva, Selvas altas y medianas perenifolias, Selva baja } \\
\text { caducifolia, Selvas húmedas, Selva mediana subperenifolia, Selva } \\
\text { tropical húmeda, Tall moist forest, Tall perennial rainforest, Tropical } \\
\text { Deciduous Forests, Tropical Dry forest, Tropical evergreen rainforest, } \\
\text { Tropical Forest semideciduous, Tropical-Forest, Tropical rainforest, } \\
\text { Tropical riparian pastures, Tropical sub-humid forest }\end{array}$ & 37 \\
\hline Bosques templados & $\begin{array}{l}\text { Bosque de encino, Bosque de pino, Bosque de pino-encino, Bosque } \\
\text { de pino asociado con matorral de coníferas (Juniperus) y de hojosas } \\
\text { (Quercus y Arbutus), Oak forests, Oakpine forests, Pine forests, Pinus } \\
\text { culminicola matorral, P. culminicola-P. hartwegii forest y P. hartwegii- } \\
\text { P. culminicola forest, Temperate forests. }\end{array}$ & 16 \\
\hline $\begin{array}{l}\text { Bosque mesófilo de } \\
\text { montaña }\end{array}$ & Bosque mesófilo de montaña. & 16 \\
\hline $\begin{array}{l}\text { Vegetación árida y } \\
\text { semiárida }\end{array}$ & $\begin{array}{l}\text { Desert shrub, matorral espinoso, matorral tamaulipeco espinoso, } \\
\text { matorral xerófilo, scrub forest dominated by Euphorbia rossiana and } \\
\text { thorn-scrub forest dominated by Dasylirion serratifolium and } \\
\text { Gochnatia hypoleuca, semiarid shrubland, semiarid zones, Sonoran } \\
\text { desert scrub and sarcocaulescent scrub, Tamaulipan thornscrub, xeric } \\
\text { scrub community dominated by Pittocaulon praecox growing on a } \\
\text { lava field }\end{array}$ & 15 \\
\hline Humedales & $\begin{array}{l}\text { Humedales costeros, humedal herbáceo de agua dulce, mangrove, } \\
\text { riparian forest }\end{array}$ & 6 \\
\hline Suelo sin vegetación & Cárcavas, coastal dunes, suelo con depósitos de cenizas & 5 \\
\hline Otros & Island, live fences, shrub & 4 \\
\hline
\end{tabular}

\section{DISCUSIÓN}

La restauración ecológica es una disciplina emergente, cuyo crecimiento en México es notorio, pero aún incipiente. En esta revisión, se evidenció un incremento en los esfuerzos desarrollados en los últimos ocho años por diferentes grupos de trabajo, quienes se han enfocado en la recuperación de ecosistemas perturbados. Sin embargo, se debe resaltar el menor porcentaje de estudios sobre REs, relacionadas, en parte, con las limitaciones logísticas de la restauración y con la complejidad inherente a la transdiciplinariedad (Carabias et al., 2007; Sarukhán, 2007; Newton, 2008; Lindig-Cisneros, 2010). En este sentido el reto es conjuntar e interrelacionar conocimientos entre grupos de trabajo de áreas tales como la ecología, antropología, sociología, economía y política, entre otras (Choi et al., 2008).

A pesar de las dificultades en realizar restauración ecológica en México, en 71\% de los estados del país, se tienen evidencias de haberse realizado esfuerzos de restauración. No hay una explicación contundente de por qué Aguascalientes, Chihuahua, Colima, Durango, Guerrero, Oaxaca, Sinaloa y Zacatecas no tengan trabajos publicados en REs. Es posible que estos estados tengan problemáticas sociales y económicas particulares, tales como la carencia de recursos que se los impidan (Carabias et al., 2007; Ceccon et al., 2015). Mientras que Chiapas y Veracruz tuvieron el mayor número de trabajos, presentando ambos altas tasas de deforestación y siendo sedes de varios centros de 
investigación especializados en el tema (Bonfil et al., 2015). Hay un sesgo en las investigaciones hacia bosques tropicales, templados y mesófilos de montaña, que es consistente con lo documentado previamente para México (Bonfil et al., 2015) y a nivel global donde $50 \%$ de las publicaciones fueron realizadas en bosques tropicales y templados (Wortley, Hero y Howes, 2013). Por otro lado, los pastizales naturales y los manglares fueron los ecosistemas con menor número de referencias, lo que es preocupante si se considera para ambos su limitada área de distribución y el alto impacto que las actividades humanas han tenido sobre ellos. Por ejemplo, los manglares han perdido 35\% de su superficie en los últimos 20 años (Flores-Verdugo et al., 2007).

En esta revisión se identificaron varios aspectos que dificultan el desarrollo de la restauración ecológica en México. Entre estos, sobresale la falta de difusión de los resultados, lo que genera un déficit de antecedentes importantes para los interesados en el área, debido a que, al no compartirse conocimientos previos y al no poderse estudiar casos aplicados en campo, se repiten errores (Carabias et al., 2007). Es así como un número importante de proyectos y experiencias de restauración no se documentan o solo se conservan como reportes técnicos, frecuentemente confidenciales y no disponibles en Internet. Lo anterior podría incrementarse como consecuencia de que recientemente consultoras $u$ organizaciones no gubernamentales obtienen financiamiento para proyectos de restauración, pero no necesariamente tienen como objetivo la publicación de sus resultados. Por lo que, aunque escasa, la información en memorias de congresos es valiosa, dado que pocas veces es publicada en otros medios.

Otra de las limitaciones encontradas fue que la mayoría de los trabajos publicados dan respuesta a pocas de las preguntas señalados por Hobbs et al. (2007) para realizar un proyecto de restauración ecológica; de modo que solo seis de 96 publicaciones cumplieron con todas. La pregunta “¿Qué restaurar?” fue la que se respondió en menor número de ocasiones. Esto muestra que el establecimiento de un ecosistema de referencia recibe poca atención en los estudios. Generalmente se debe a la falta de información sobre el sitio o de las referencias históricas que permitan conocer las características bióticas y abióticas del ecosistema antes de los disturbios.
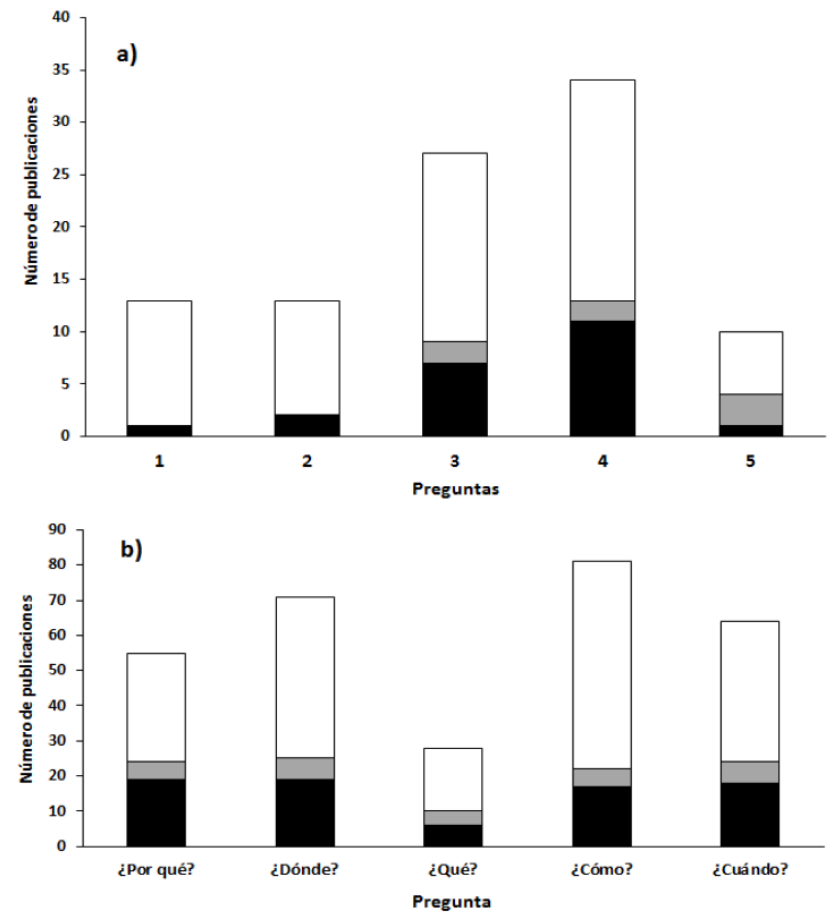

FIGURA 4. Número de publicaciones (1999-2016) para: a) que responden entre una y cinco de las preguntas de investigación sobre restauración ecológica (sensu Hobbs et al., 2007); b) que contestan preguntas particulares de investigación sobre restauración ecológica (sensu Hobbs et al., 2007).

En negro se indican los trabajos de restauración ecológica sensu stricto; en blanco, los de ecología de la restauración y en gris, los trabajos que incluyen objetivos de ambos. Se excluye información de congresos.

Se resalta que la selección de un ecosistema de referencia debe enfocarse en la obtención de un sistema funcional a largo plazo y no necesariamente en la recuperación del ecosistema antes del disturbio (Hobbs et al., 2007). En general, la restauración ecológica no considera la recuperación de todas las dinámicas del ecosistema que ya se han perdido (Reis, Campanhã y Regina, 2010). De este modo, se propone que al definir el ecosistema de referencia se busque obtener a un sistema funcional, aunque este difiera estructuralmente de los ecosistemas vecinos (Shackelford et al., 2013). Recientemente, se ha señalado la escala de paisaje para la restauración, donde se propone un índice para la selección de especies útiles en la restauración de suelos agrícolas, considerándolos como zonas clave en los procesos de sucesión vegetal (Martínez-Ramos et al., 2016).

La mayoría de las publicaciones, por su parte, contestan a la pregunta “¿Cuándo restaurar?”. Esta cuestión considera los esfuerzos por manejar el sitio antes de la restauración. Por ejemplo, proponen tomar medidas de protección y recuperación de los ecosistemas con el fin de evitar que su deterioro llegue a 
un nivel en el que la única solución sea la restauración activa. Asimismo, proponen estrategias que faciliten el establecimiento de especies sucesionales, intermedias y tardías (López et al., 2010; Meli y Dirzo, 2013; Meli, Rey-Benayas, Martínez-Ramos y Carabias, 2015; Rangel-Landa, Casas y Dávila, 2015). En esta pregunta se toma en cuenta el desarrollo y crecimiento de las especies seleccionadas, sin embargo, esta suele ser una de las principales problemáticas a las que se enfrentan los proyectos de restauración, debido a la falta de viveros en las comunidades elegidas. El apoyo tanto económico como de las comunidades para esta etapa es un punto indispensable, por lo que se deben conjuntar esfuerzos en el área (Shackelford et al., 2013). Además, hay que tomar en cuenta que los objetivos y estrategias de restauración deberían irse ajustando según los resultados que se vayan obteniendo a lo largo del proyecto (Zaldívar-Jiménez, Guevara-Porras, Pérez-Ceballos, Díaz-Mondragón y RosadoSolórzano, 2017).

Como parte fundamental de un proyecto de restauración ecológica se destaca el monitoreo a largo plazo, con el fin de corroborar que se lograron los resultados esperados (aún después de años de terminado el estudio). En pocos de los trabajos revisados se incluyeron aspectos de monitoreo, de modo que es difícil evaluar el resultado de los proyectos. Además, no es posible asegurar que los recursos naturales, económicos y humanos utilizados durante el proceso de restauración se mantendrán por más tiempo y, por lo tanto, los esfuerzos pueden perderse en poco tiempo. Junto al periodo de monitoreo, el plazo de establecimiento de las plantas puede ser largo (Vargas, 2007), lo que complica la obtención de financiamientos que brinden recursos suficientes para un proyecto de restauración completo (Carabias et al., 2007). La importancia del monitoreo permite, entre otras cosas, evaluar el éxito del proyecto. Li, Cadotte, Martínez- Garza, la Peña- Domene y Du (2017) observan, después de ocho años de monitoreo, que la restauración en bosques tropicales con especies dispersadas por viento y animales, permite el establecimiento de grupos filogenética y funcionalmente relacionados, señalando el avance de la regeneración vegetal. Sin embargo, en general se ha optado por estudios cortos y con mayor enfoque teórico que práctico, es decir, en el ámbito de la ecología de la restauración.
La extensión territorial necesaria para la restauración también es una limitante, debido a la gran diversidad cultural y a los problemas sociales, económicos y políticos que disminuyen las áreas para restaurar (González-Espinosa et al., 2007; Ceccon et al., 2015). De este modo, al requerirse de periodos prolongados de tiempo para llevarse a cabo la restauración, se inhabilita la posibilidad del uso del suelo por los dueños, minimizando sus ganancias económicas por lago tiempo. Esto reduce la posibilidad de obtener extensiones territoriales grandes para restaurar y, como consecuencia, los estudios se llevan a cabo en parcelas experimentales de tamaño reducido. Por lo anterior, la selección de los sitios a restaurar debe llevarse a cabo con la participación activa de los dueños de las tierras. Sin embargo, para logarlo, se necesita un fuerte trabajo de vinculación y gestión, con el objetivo de que los pobladores locales se adueñen del proyecto, lo cual es difícil, al considerar que la mayoría de los profesionales no se encuentran entrenados para trabajar con grupos sociales (Sarukhán, 2007). Además, se resalta la necesidad de la inclusión de los conocimientos tradicionales como base de las investigaciones, de modo que se conjunten con los conocimientos científicos para la conservación de sistemas megadiversos y multiculturales (Allen, Allen y Gómez-Pompa, 2005; Alanís-Rodríguez et al., 2010; Lindig-Cisneros, 2010).

El establecimiento de técnicas para la restauración o el “Cómo restaurar?" fue la pregunta que mostró una mayor atención en la bibliografía. En este sentido, sobresale el metaanálisis realizado por Ceccon, González y Martorell (2016), en que puntualizan que la siembra directa de las semillas en el sitio a restaurar no es método efectivo, al perderse prácticamente todos los individuos. Otra deficiencia aún notoria es la ausencia de estudios que cuantifiquen los costos de la restauración. Esta es una de las problemáticas más importantes, debido a que en la mayoría de los proyectos de recuperación de ecosistemas se buscan resultados rápidos y económicos, características que no se observan a primera vista en la restauración, incluso cuando esta es activa. Por esta razón, se agradece que algunos trabajos, tales como los de Allen et al. (2005), Guevara, Laborde y Sánchez-Ríos (2005) y Zaldívar-Jiménez, Guevara-Porras, Pérez-Ceballos, Díaz-Mondragón y Rosado-Solórzano (2010), dediquen parte de su estudio a proponer estrategias poco costosas y sencillas de realizar en los proyectos de restauración, o el de Douterlungne et 
al. (2015) quienes contabilizaron los gastos requeridos para diferentes técnicas de restauración. Además, se valoran estudios que brindan propuestas o consejos que faciliten el proceso, como los estudios de Badano, Pérez y Vergara (2009), quienes ofrecen una propuesta de cuatro etapas para la regeneración de zonas secas o el de Zaldívar-Jiménez et al. (2010), quienes proponen cinco etapas para la recuperación de humedales.

Las preguntas “¿Por qué y dónde restaurar?" fueron tomadas en cuenta por más de la mitad de las publicaciones, lo cual se debe a que estas preguntas se centran en brindar justificaciones para la restauración del sitio. Estudios recientes resaltan la importancia de la pregunta “¿Por qué restaurar?", al ser clave en el desarrollo de los objetivos de la restauración (Martin, 2017). En este sentido, la mayoría de la literatura justificó la selección del sitio (incluyendo las perturbaciones y problemáticas en la zona), de modo que los objetivos (y futuros resultados) se enfocaron en las características propias de cada sitio. La descripción general de la zona a restaurar, que incluye el tipo de vegetación, especies dominantes, localización, extensión, características abióticas y estado sucesional, también brinda información clave para el desarrollo de la restauración. A pesar de que los estudios muestran una tendencia a definir el sitio a restaurar, en ningún trabajo se pudo observar la búsqueda por la restauración ecológica a través de corredores, que permitan la conexión entre sitios restaurados y no restaurados. Por lo anterior se ha recomendado la reflexión de esto como un punto clave en la recuperación del paisaje (Shackelford et al., 2013).

La mayoría de las publicaciones revisadas incluyeron enunciados donde se destacó la múltiple participación en la toma decisiones por parte del sector social, los centros de investigación científica, las asociaciones gubernamentales y las no gubernamentales. Esto es destacable, en el tenor de que se comparten objetivos comunes, incluso en la repartición de apoyos económicos (Lindig-Cisneros, 2010). El financiamiento es un factor crucial para desarrollar proyectos exitosos de restauración, sin embargo, son escasas las fuentes, siendo probablemente la Comisión Nacional para el Conocimiento y Uso de la Biodiversidad [Conabio] y la Secretaria del Medio Ambiente y Recursos Naturales [Semarnat], las únicas instituciones federales que destinan recursos para este fin. Además, un considerable porcentaje de recursos federales es destinado a la Conafor para realizar proyectos de reforestación que, aunque cumplen con ciertos objetivos al incrementar la cobertura forestal, no implican restauración ecológica. Además, falta mucho interés por parte de los gobiernos estatales en el tema y mucha gestión para obtener fondos internacionales. Así, el financiamiento es el aspecto más importante para el desarrollo de la restauración ecológica en México.

El número de estudios publicados, encontrados y revisados solo con respecto a REs fue bajo. El reto futuro será incrementar la ejecución de proyectos y publicar resultados. Además, será necesario adecuarse al hecho de que la restauración es dinámica (Perring et al., 2015; Martin, 2017), enfrentando los constantes cambios a los que se enfrenta el planeta, incluyendo el cambio climático (Gelviz-Gelvez, Pavón, Illoldi-Rangel y BallesterosBarrera, 2014). La restauración ecológica es una tarea transdiciplinaria que conlleva a la inclusión de múltiples actores, tanto de la academia, la política y la propia ciudadanía, quienes conviven dentro de un sistema socioecológico complejo y dinámico (Choi et al., 2008; Perring et al., 2015).

\section{CONCLUSIONES}

En México la restauración ecológica es una disciplina emergente, la cual, a pesar de las complicaciones políticas, económicas y sociales del país, ha ido surgiendo lentamente, recibiendo cada vez mayor atención por diferentes grupos de trabajo. Aunque se obtuvieron pocos estudios de REs, sí se apreció un incremento de estos en los últimos 10 años. Sin embargo, hay un sesgo hacía selvas y bosques templados, descuidando otros ecosistemas importantes como son los pastizales y humedales. Analizar los trabajos desde la perspectiva propuesta por Hobbs et al. (2007) permitió identificar las carencias u omisiones en la información que proporcionan los proyectos, en particular sobre "qué es lo que se quiere restaurar", es decir, la visión de la restauración. Esto debe ser básico para plantear las metodologías y seleccionar las especies. Finalmente, en esta revisión se destaca la necesidad de generar equipos multidisciplinarios, para cubrir con los aspectos necesarios y generar proyectos de restauración ecológica dentro de un sistema socioecológico dinámico. 


\section{REFERENCIAS}

Alanís-Rodríguez, E., Jiménez-Pérez, J., Pando-Moreno, M., AguirreCalderón, Ó. A., Treviño-Garza, E. J., \& García-Galindo, P. C. (2010). Efecto de la restauración ecológica post-incendio en la diversidad arbórea del Parque Ecológico Chipinque, México. Madera y bosques, 16(4), 39-54. doi:10.21829/myb.2010.1641159

Allen, M. F., Allen, E. B., \& Gómez- Pompa, A. (2005). Effects of mycorrhizae and nontarget organisms on restoration of a seasonal tropical forest in Quintana Roo, Mexico: factors limiting tree establishment. Restoration Ecology, 13(2), 325-333. doi:10.1111/j.1526-100X.2005.00041.x

Armesto, J. J., Bautista, S., Del Val, E., Ferguson, B., García, X., Gaxiola, A., Godinez-Álvarez, H., Gann, G., López-Barrera, F., Manson, R., Núñez-Ávila, M., Ortiz-Arrona, C., Tognetti, P., \& WilliamsLinera, G. (2007). Towards in ecological restoration network: Reversing land degradation in Latin America. Frontiers in Ecology and the Environment, 5(4), 1-4. doi:10.1890/15409295(2007)5[w1:TAERNR]2.0.CO;2

Badano, E. I., Pérez, D., \& Vergara, C. H. (2009). Love of nurse plants is not enough for restoring oak forests in a seasonally dry tropical environment. Restoration Ecology, 17(5), 571-576. doi:10.1111/j.1526-100X.2009.00530.x

Bonfil, C., Fernández y Fernández, D., \& González-Espinosa, M. (2015). Un análisis preliminar del estado actual de los estudios de restauración ecológica en México. En B. Brow (Ed.), Tzintzuntzan, el lugar de los colibries - otra vez / Tzintzuntzan, place of the bummingbirds - again. Museo de Arte Contemporáneo Alfredo Zalce, Morelia (p. 28-39). Morelia, Michoacán: Editorial Estampas.

Carabias, J., Arriaga, V., \& Cervantes-Gutiérrez, V. (2007). Las políticas públicas de la restauración ambiental en México: limitantes, avances, rezagos y retos. Boletín de la Sociedad Botánica de México, 80(Suplemento), 85-100. doi:10.17129/botsci.1759

Ceccon, E., Barrera- Cataño, J. I., Aronson, J., \& Martínez- Garza, C. (2015). The socioecological complexity of ecological restoration in Mexico. Restoration Ecology, 23(4), 331-336. doi:10.1111/rec.12228

Ceccon, E., González, E. J., \& Martorell, C. (2016). Is direct seeding a biologically viable strategy for restoring forest ecosystems? Evidences from a Meta- analysis. Land Degradation \& Development, 27(3), 511-520. doi:10.1002/ldr.2421

Choi, Y. D., Temperton, V. M., Allen, E. B., Grootjans, A. P., Halassy, M., Hobbs, R. J., Naeth, M. A., \& Torok, K. (2008). Ecological restoration for future sustainability in a changing environment. Ecoscience, 15(1), 53-64. https://doi.org/10.2980/11956860(2008)15[53:ERFFSI]2.0.CO;2
Comisión Nacional Forestal [Conafor], \& Universidad Autónoma Chapingo [UACh] (2013). Línea Base Nacional de degradación de tierras y desertificación. Informe final. Zapopan, México: Comisión Nacional Forestal y Universidad Autónoma Chapingo.

Douterlungne, D., Ferguson, B. G., Siddique, I., Soto- Pinto, L., Jímenez-Ferrer, G., \& Gavito, M. E. (2015). Microsite determinants of variability in seedling and cutting establishment in tropical forest restoration plantations. Restoration Ecology, 23(6), 861-871. doi:10.1111/rec.12247

Flores-Verdugo, F., Moreno-Casasola, P., Agraz-Hernández, C. M., López-Rosas, H., Benítez-Pardo, D., \& Travieso-Bello, A. C. (2007). La topografía y el hidroperiodo: dos factores que condicionan la restauración de humedales costeros. Boletín de la Sociedad Botánica de México, 80(Suplemento), 33-47.

Gelviz-Gelvez, S. M., Pavón, N. P., Illoldi-Rangel, P., \& BallesterosBarrera, C. (2014). Ecological niche modeling under climate change to select shrubs for ecological restoration in Central Mexico. Ecological Engineering, 74, 302-309. doi:10.1016/j.ecoleng.2014.09.082

González-Espinosa, M., Ramírez-Marcial, N., Camacho-Cruz, A., Holz, S. C., Rey-Benayas, J. M., \& Parra-Vázquez, M. R. (2007). Restauración de bosques en territorios indígenas de Chiapas: modelos ecológicos y estrategias de acción. Boletín de la sociedad Botánica de México, 80(Suplemento), 11-23. doi:10.17129/botsci.1753

Guevara, S., Laborde, J., \& Sánchez-Ríos, G. (2005). Los árboles que la selva dejó atrás. Interciencia, 30(10), 595-601.

Hobbs, R. J., Walker L. R., \& Walker, J. (2007). Integrating restoration and succession. En L. R. Walker, J. Walker, \& R. J. Hobbs (Eds.), Linking restoration and ecological succession (p. 168-179). Londres, Inglaterra: Springer.

Li, L., Cadotte, M. W., Martínez- Garza, C., la Peña- Domene, M., \& Du, G. (2017). Planting accelerates restoration of tropical forest but assembly mechanisms appear insensitive to initial composition. Journal of Applied Ecology. doi:10.1111/13652664.12976

Lindig-Cisneros, R. (2010). Ecological restoration in Mexico: The challenges of a multicultural megadiverse country. Ecological Restoration, 28(3), 232-233. doi:10.1353/ecr.2010.0033

López, H., López-Barrera, F., Moreno-Casasola, P., Aguirre-León, G., Cázares-Hernández, E., \& Sánchez-Higueredo, L. (2010). Indicators of recovery in a Tropical Freshwater Marsh invaded by an african grass. Ecological Restoration, 28(3), 324-332. doi:10.3368/er.28.3.324 
López, L. (2016). Supervivencia y crecimiento de Pinus gregii Engelm, ex Parl, en reforestaciones establecidas entre 2000 y 2012 en ambientes contrastantes en el estado de Hidalgo. Tesis de maestría no publicada, Universidad Autónoma del Estado de Hidalgo, México.

Martin, D. M. (2017). Ecological restoration should be redefined for the twenty- first century. Restoration Ecology. doi:10.1111/rec.12554

Martínez-Ramos, M., Pingarroni, A., Rodríguez-Velázquez, J., Toledo- Chelala, L., Zermeño- Hernández, I., \& Bongers, F. (2016). Natural forest regeneration and ecological restoration in human- modified tropical landscapes. Biotropica, 48(6), 745-757. doi:10.1111/btp.12382

Meli, P., \& Dirzo, R. (2013). Effects of grasses on sapling establishment and the role of transplanted saplings on the light environment of pastures: implications for tropical forest restoration. Applied Vegetation Science, 16(2), 296-304. doi:10.1111/j.1654109X.2012.01217.x

Meli, P., Rey-Benayas, J. M. R., Martínez-Ramos, M., \& Carabias, J. (2015). Effects of grass clearing and soil tilling on establishment of planted tree seedlings in tropical riparian pastures. New Forests, 46(4), 507-525. doi:10.1007/s11056-015-9479-3

Newton, A. C. (2008). Restoration of dryland forests in Latin America: The ReForLan project. Ecological Restoration, 26(1), 10-13. doi:10.3368/er.26.1.10

Organización de las Naciones Unidas para la Alimentación y la Agricultura [FAO]. (2010). Evaluación de los recursos forestales mundiales 2010, Informe principal. Montes Roma, Italia: Estudio FAO.

Organización de las Naciones Unidas para la Alimentación y la Agricultura [FAO]. (2015). Evaluación de los recursos forestales mundiales 2015. ¿Cómo están cambiando los bosques del mundo? Compilación de datos. Roma, Italia: FAO.

Perring, M. P., Standish, R. J., Price, J. N., Craig, M. D., Erickson, T. E., Ruthrof, K. X., Whiteley, A. S., Valentine, L. E., \& Hobbs, R. J. (2015). Advances in restoration ecology: rising to the challenges of the coming decades. Ecosphere, 6(8), 1-25. doi:10.1890/ES1500121.1

Porter-Bolland, L., Ellis, E. A., Guariguata, M. R., Ruiz-Mallén, I., Negrete-Yankelevich, S., \& Reyes-García, V. (2011). Community managed forests and forest protected areas: An assessment of their conservation effectiveness across the tropics. Forest ecology and management, 268, 6-17. doi:10.1016/j.foreco.2011.05.034

Rangel-Landa, S., Casas, A., \& Dávila, P. (2015). Facilitation of Agave potatorum: An ecological approach for assisted population recovery. Forest Ecology and Management, 347, 57-74. doi:10.1016/j.foreco.2015.03.003
Reis, A., Campanhã, F., \& Regina, D. (2010). Nucleation in tropical ecological restoration. Scientia Agricola, 67(2), 244-250. doi:10.1590/S0103-90162010000200018

Sarukhán, J. (2007). Editorial. Boletin de la Sociedad Botánica de México, 80(Suplemento), 7-10.

Shackelford, N., Hobbs, R. J., Burgar, J. M., Erickson, T. E., Fontaine, J. B., Laliberté, E., Ramalho, C. E., Perring, M. P., \& Standish, R. J. (2013). Primed for change: developing ecological restoration for the 21st century. Restoration Ecology, 21(3), 297-304. doi:10.1111/rec.12012

Society for Ecological Restoration [SER] (2004). SER International Primer on Ecological Restoration. Recuperado de http://www.ser.org/resources/resources-detail-view/serinternational-primer-on-ecological-restoration.

Vargas, O. (Ed.). (2007). Guía Metodológica para la restauración ecológica del bosque altoandino. Bogotá, Colombia: Universidad Nacional de Colombia.

Wortley, L., Hero, J. M., \& Howes, M. (2013). Evaluating ecological restoration success: a review of the literature. Restoration Ecology, 21(5), 537-543. doi:10.1111/rec.12028

Zaldívar-Jiménez, M. A., Herrera-Silveira, J. A., Teutli-Hernández, C., Comín, F. A., Andrade, J. L., Molina, C. C., \& Ceballos, R. P. (2010). Conceptual framework for mangrove restoration in the Yucatán Peninsula. Ecological Restoration, 28(3), 333-342. doi:10.3368/er.28.3.333

Zaldívar-Jiménez, A., Guevara-Porras, P., Pérez-Ceballos, R., DíazMondragón, S., \& Rosado-Solórzano, R. (2017). US-Mexico joint Gulf of Mexico large marine ecosystem based assessment and management: Experience in community involvement and mangrove wetland restoration in Términos lagoon, Mexico. Environmental Development, 20, 206-213. doi:10.1016/j.envdev.2017.02.007

Manuscrito recibido el: 16 de Agosto de 2016

Aceptado el: 6 de septiembre de 2017

Publicado el: 9 de marzo de 2018

Este documento se debe citar como:

Calva-Soto, K., \& Pavón, N. P. (2O18). La restauración ecológica en México: una disciplina emergente en un país deteriorado. Madera $y$ Bosques, 24(1), 2411135. doi: 10.21829/myb.2018.2411135

Madera y Bosques por Instituto de Ecología, A.C. se distribuye bajo una Licencia Creative Commons Atribución-NoComercialCompartirlgual 4.0 Internacional. 
Calva-Soto y Pavón. La restauración ecológica: una disciplina emergente en un país deteriorado 